Pacific Journal of Mathematics

GENERALIZED PRIMITIVE ELEMENTS FOR
TRANSCENDENTAL FIELD EXTENSIONS 


\title{
GENERALIZED PRIMITIVE ELEMENTS FOR TRANSCENDENTAL FIELD EXTENSIONS
}

\author{
JAMES K. DEVEnEY
}

Let $L$ be a finitely generated separable extension of a field $K$ of characteristic $p \neq 0$. Artin's theorem of a primitive element states that if $L$ is algebraic over $K$, then $L$ is a simple extension of $K$. If $L$ is non-algebraic over $K$, then an element $\theta \in L$ with the property $L=L^{\prime}(\theta)$ for every $L^{\prime}, L \supseteq L^{\prime} \supseteq K$, such that $L$ is separable algebraic over $L^{\prime}$ is called a generalized primitive element for $L$ over $K$. The main result states that if $\left[K: K^{p}\right]>$ $p$, then there exists a generalized primitive element for $L$ over $K$. An example is given showing that if $\left[K: K^{p}\right] \leqq p$, then $L$ need not have a generalized primitive element over $K$.

I. Introduction. Let $L$ be a finitely generated extension of a field $K$ of characteristic $p \neq 0$. Artin's theorem of the primitive element states that if $L$ is separable algebraic over $K$, then $L$ is a simple extension of $K$. In this paper we examine the following analogue of Artin's theorem in the case where $L$ is a separable non-algebraic extension of $K$. Does there exist an element $\theta \in L$ with the property that $\theta$ is a primitive element for $L$ over every intermediate field $L^{\prime}$ such that $L$ is separable algebraic over $L^{\prime}$ ? The main result states that if $K$ has at least two elements in a $p$-basis, then there does exist such a generalized primitive element (Theorem 4). Such elements $\theta$ are characterized by the condition that $L$ is reliable over $K(\theta)$ (Theorem 1). As a corollary, it follows that automorphisms of $L$ over $K$ are uniquely determined by their action on a generalized primitive element $\theta$. Other results which indicate the essential nature of a generalized primitive element include the following. If $L_{1}$ and $L_{2}$ are intermediate fields of $L / K$ where $L$ is separable over $L_{1}$ and $L_{2}$, then $L_{2} \supseteq L_{1}$ if and only if some generalized primitive element for $L_{1}$ is in $L_{2}$ (Theorem 6).

II. Generalized primitive elements. Throughout we assume $L$ is a finitely generated extension of a field $K$ of characteristic $p \neq 0$. As usual, a relative $p$-basis for $L$ over $K$ is a minimal generating set for $L$ over $K\left(L^{p}\right)$.

Definition. $\quad L$ is a reliable extension of $K$ if $L=K(M)$ for every relative $p$-basis $M$ of $L$ over $K$. 
In the case where $L$ is finitely generated over $K, L$ is reliable over $K$ if and only if there does not exist a proper intermediate field $L^{\prime}$ with $L$ separable over $L^{\prime}$ [5, Theorem 1, p. 524]. Using this result it follows that if $L$ is reliable over $K$, then $L$ is reliable over any intermediate field $M$.

THEOREM 1 [1, Theorem 1.9]. If $L$ is finitely generated over $K$, then there exists a unique intermediate field $C$ with the property $L / C$ is separable and $C / K$ is reliable.

In fact, $C$ is the intersection of all subfields $L^{\prime}$ such that $L / L^{\prime}$ is separable. If $L$ is separable over $K$, then an element $\theta$ in $L$ is a generalized primitive element for $L$ over $K$ if $L=L^{\prime}(\theta)$ for any $L^{\prime}$ such that $L$ is separable algebraic over $L^{\prime}$. Henceforth, $L$ will be a finitely generated separable (non-algebraic) extension of $K$.

THEOREM 2. An element $\theta$ in $L$ is a generalized primitive element for $L$ over $K$ if and only if $L$ is reliable over $K(\theta)$.

Proof. Assume $\theta$ is a generalized primitive element. It suffices to show there are no proper intermediate fields $L^{\prime}, L \supset L^{\prime} \supseteq K(\theta)$, over which $L$ is separable. Since $\theta$ is a generalized primitive element, there are no proper fields over which $L$ is separable algebraic. But in any finitely generated separable extension $L / L^{\prime}$ there exist subfields over which $L$ is separable algebraic (by applying Luroth's Theorem). Thus $L / K(\theta)$ is reliable.

Conversely, assume there exists an element $\theta$ such that $L$ is reliable over $K(\theta)$ and let $L^{\prime}$ be any intermediate field such that $L / L^{\prime}$ is separable algebraic. Then $L / L^{\prime}(\theta)$ is also separable. Since $L / K(\theta)$ is reliable and $L^{\prime} \supseteq K, L / L^{\prime}(\theta)$ is reliable and hence $L=L^{\prime}(\theta)$.

The following result of Mordeson and Vinograde is essential to this paper.

Theorem 3 [4, Theorem 2]. Assume $L$ is a finitely generated separable extension of $K, L \neq K$, and assume $\left[K: K^{p}\right]>p$. Then there exists a field $M=L(\alpha)$ where $M$ is reliable over $K$ and $\alpha^{p}$ is in $L$.

THEOREM 4. Let $L$ be a finitely generated separable extension of $K$ and assume $\left[K: K^{p}\right]>p$. Then there exists a generalized primitive element for $L$ over $K$.

Proof. By Theorem 3, there exists a field $M=L(\alpha)$ which is reliable over $K$ and $\alpha^{p} \in L$. Let $\theta=\alpha^{\rho}$ and we show $\theta$ is the desired element. By Theorem 2, it suffices to show $L$ is reliable over $K(\theta)$. Assume there exists an intermediate field $L^{\prime}, L \supseteq L^{\prime} \supseteq K(\theta)$ 
where $L$ is separable over $L^{\prime}$. Since $\alpha^{p} \in K(\theta), \alpha^{p} \in L^{\prime}$. Thus $L^{\prime}(\alpha)$ is purely inseparable over $L^{\prime}$. Thus $L$ and $L^{\prime}(\alpha)$ are linearly disjoint over $L^{\prime}$. By [3, Corollary 4, p. 265], $L^{\prime}(\alpha)(L)=M$ is separable over $L^{\prime}(\alpha)$. As $M$ is reliable over $L^{\prime}(\alpha), M=L^{\prime}(\alpha)$ and since $L$ and $L^{\prime}(\alpha)$ are linear disjoint over $L^{\prime}$, we must have $L=L^{\prime}$ and $L$ is reliable over $K(\theta)$.

COROllary 1. If $L / K$ is nonalgebraic, then any generalized primitive element is transcendental over $K$.

Proof. Let $\theta$ be a generalized primitive element. If $\theta$ were algebraic over $K$, then $L / K(\theta)$ would be separable and hence $L=K(\theta)$.

The following corollary is a direct result of a calculation in [4]. For completeness, it is presented here.

Corollary 2. Assume $L=K\left(z_{1}, \cdots, z_{n-1}, z_{n}\right)$ where $z_{1}, \cdots, z_{n-1}$ are algebraically independent over $K$ and $F / K\left(z_{1}, \cdots, z_{n-1}\right)$ is nontrivial separable. Let $\{x, y\}$ be $p$-independent in $K$. Then $\theta=\alpha^{p}$ is a generalized primitive element for $L / K$ where

$$
\alpha=\sum_{1}^{n-1} k_{j} z_{j}^{p^{i}}+k_{n} z_{n}^{p^{n-1}}
$$

and

$$
\begin{gathered}
k_{1}=y^{-1} \\
k_{l}=(-1)^{j-1} \frac{x^{p^{0+\cdots+p^{j-2}}}}{y^{p^{0+\cdots+p^{j-1}}}} \text { for } j=2, \cdots, n-1 \\
k_{n}=(-1)^{n-1}\left(\frac{x}{y}\right)^{p^{0}+\cdots+p^{n-2}} .
\end{gathered}
$$

Proof. This follows from Theorem 4 and the proof of $[4$, Theorem 1, p. 44].

COROLlARY 3. Let $\theta$ be a generalized primitive element for $L$ over $K$. Then any automorphism of $L$ over $K$ is uniquely determined by its action on $\theta$.

Proof. Let $\sigma, \tau$ be automorphisms of $L / K$ and assume $\sigma(\theta)=$ $\tau(\theta)$. Then $\sigma \tau^{-1}(\theta)=\theta$ and $K(\theta)$ is contained in the fixed field $L^{\prime}$ of $\sigma \tau^{-1}$. Since $L$ is separable over $L^{\prime}, L=L^{\prime}$ and $\sigma=\tau$. 
LEMMA 1. Let $\theta$ be a generalized primitive element for $L$ over $K$, and let $F$ be an intermediate field such that $L$ is separable nonalgebraic over $F$. Then $F$ is free from $K(\theta)$ and $F(\theta)$ is separable over $K(\theta)$.

Proof. If $\theta$ were algebraic over $F$, then $L$ would be separable over $F(\theta)$, a contradiction to $L$ being reliable over $F(\theta)$. Thus $K(\theta)$ is free from $F$. The remainder of the Lemma follows from [3, Corollary $4, \mathrm{p}$. 265].

A generalized primitive element for $L$ over $K$ will generate $L$ over any subfield $L^{\prime}$ such that $L$ is separable algebraic over $L^{\prime}$. The following theorem shows that with one exception these are the only subfields with this property.

THEOREM 5. Let $\theta$ be a generalized primitive element for $L$ over $K$, and let $L^{\prime}$ be a subfield of $L$ containing $K$. Then $L=L^{\prime}(\theta)$ if and only if either $L / L^{\prime}$ is separable algebraic or $L=K(\theta)$.

Proof. Assume $L=L^{\prime}(\theta)$. If $L / L^{\prime}$ are not algebraic, then $L / L^{\prime}$ would be pure transcendental and hence separable. But then by Lemma $1, L / K(\theta)$ would be separable, and hence $L=K(\theta)$. Thus we may assume $L / L^{\prime}$ is algebraic and $L \neq K(\theta)$. Since $L / K(\theta)$ is not separable and $L / K$ is, $\theta \in K\left(L^{p}\right)$ [1, Proposition 1.3]. Thus $L=L^{\prime}\left(L^{p}\right)$ and $L$ is relatively perfect over $L^{\prime}$. Since $L / L^{\prime}$ is also finitely generated, $L / L^{\prime}$ is separable algebraic [6, Theorem 2, p. 419]. The converse is Theorem 2.

If $L$ is a finitely generated separable extension of $K$, then any intermediate field $L^{\prime}$ is also finitely generated and separable over $L$. If $\left[K: K^{p}\right]>p$, then $L^{\prime}$ will also have a generalized primitive element $\theta^{\prime}$ over $K$. Moreover, each element of $L$ will be a generalized primitive element for a unique subfield $L^{\prime}$ where $L / L^{\prime}$ is separable. For if $\theta \in L$, let $L^{\prime}$ be the unique intermediate field of $L / K(\theta)$ such that $L$ is separable over $L^{\prime}$ and $L^{\prime}$ is reliable over $K(\theta)$. Then $\theta$ is a generalized primitive element for $L^{\prime}$. Thus any intermediate field $L^{\prime}$ where $L$ is separable over $L^{\prime}$ is uniquely determined by any of its generalized primitive elements. The following theorem and corollary indicate how a generalized primitive element is basic in the structure of an intermediate field.

THEOREM 6. Assume $L$ is a finitely generated separable extension of $K$ and let $L_{1}$ and $L_{2}$ be two intermediate fields over which $L$ is separable. Then the following are equivalent.

(1) $L_{1} \subseteq L_{2}$

(2) Every generalized primitive element for $L_{1}$ is in $L_{2}$

(3) Some generalized primitive element for $L_{1}$ is in $L_{2}$. 
Proof. We show (3) implies (1). Let $\theta_{1}$ be a generalized primitive element for $L_{1} / K$ and assume $\theta_{1} \in L_{2}$. If $L_{2}$ is separable over $K\left(\theta_{1}\right)$, then $L$ is separable over $K\left(\theta_{1}\right)$ and $L_{1}$ is separable over $K\left(\theta_{1}\right)$. Since $L_{1}$ is reliable over $K\left(\theta_{1}\right), L_{1}=K\left(\theta_{1}\right)$ and $L_{1} \subseteq L_{2}$. If $L_{2}$ is inseparable over $K\left(\theta_{1}\right)$, then there is a unique field $C_{2}, L_{2} \supseteq C_{2} \supseteq K\left(\theta_{1}\right)$ where $L_{2}$ is separable over $C_{2}$ and $C_{2}$ is reliable over $K\left(\theta_{1}\right)$. Thus $L$ is separable over $C_{2}$ and $C_{2}$ is reliable over $K\left(\theta_{1}\right)$. But $L_{1}$ is uniquely determined by these properties and hence $C_{2}=L_{1}$ and $L_{1} \subseteq L_{2}$.

CoROllary 4. Assume $L$ is separable over $L_{1}, L \supseteq L_{1} \supseteq K$, and $\theta_{1}$ is a generalized primitive element for $L_{1}$ over $K$. If $L_{2}$ is any intermediate field of $L$ over $K$ such that $L$ is separable algebraic over $L_{2}$, then $L_{2}\left(L_{1}\right)=L_{2}\left(\theta_{1}\right)$.

Proof. Since $L$ is separable algebraic over $L_{2}, L$ is separable over $L_{2}\left(\theta_{1}\right)$. By Theorem 6, $L_{2}\left(\theta_{1}\right) \supseteq L_{1}$ and hence $L_{2}\left(L_{1}\right)$. Obviously $L_{2}\left(\theta_{1}\right) \subseteq L_{1}\left(L_{2}\right)$ and thus $L_{2}\left(L_{1}\right)=L_{2}\left(\theta_{1}\right)$.

EXAMPLE 1. If $\left[K: K^{p}\right] \leqq p$, then $L$ may not have a generalized primitive element over $K$. Let $K$ be a perfect field and let $L=$ $K(x, y, z)$ where $\{x, y, z\}$ is algebraically independent over $K$. We claim there is no generalized primitive element for $L$ over $K$. Assume $\theta$ is one. Then $L / K(\theta)$ is reliable. However $K(\theta)$ has one element in a relative $p$-basis and hence by [2, Theorem 7 (iv)] $L$ is separable over $(K(\theta)) * \cap L$, where $(K(\theta)) *$ is the perfect closure of $K(\theta)$. But $(K(\theta)) * \cap L$ is of transcendence degree at most 1 over $K$, and hence $(K(\theta)) * \cap L \neq L$. This contradicts $L$ being reliable over $K(\theta)$.

\section{REFERENCES}

1. J. Deveney and J. Mordeson, Subfields and invariants of inseparable field extensions, preprint.

2. N. Heerema and H. F. Kreimer, Modularity vs. separability for field extensions, Canad. J. Math., 27 (1975), 1176-1182.

3. S. Lang, Algebra, Addison-Wesley, Reading, Mass., 1967.

4. J. Mordeson and B. Vinograde, Inseparable embeddings of separable transcendental extensions, Arc H. Math., 27 (1976), 42-47.

5. - Relatively separated transcendental field extensions, Arch. Math., 24 (1973), 521-526.

6. _ Separating p-bases and transcendental extension fields, Proc. Amer. Math. Soc., 31 (1972), 417-422.

Received January 20, 1976.

VIRGINIA COMMONWEALTH UNIVERSITY

RICHMOND, VA 23284 




\section{Pacific Journal of Mathematics}

Vol. 68, No. 1

March, 1977

Richard Julian Bagby, On $L^{p}, L^{q}$ multipliers of Fourier transforms . .......

Robert Beauwens and Jean-Jacques Van Binnebeek, Convergence theorems in

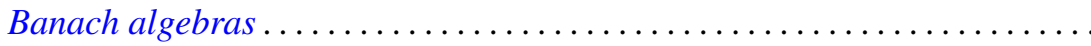

James Cyril Becker, Skew linear vector fields on spheres in the stable

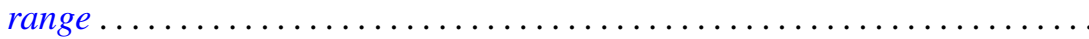

Michael James Beeson, Continuity and comprehension in intuitionistic formal

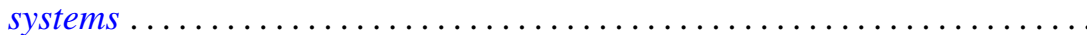

James K. Deveney, Generalized primitive elements for transcendental field

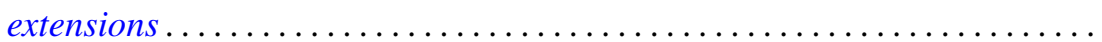

Samuel S. Feder, Samuel Carlos Gitler and K. Y. Lam, Composition properties

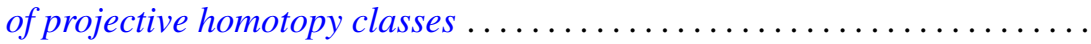

Nathan Jacob Fine, Tensor products of function rings under composition ......

Benno Fuchssteiner, Iterations and fixpoints . . . . . . . . . . . . . .

Wolfgang H. Heil, On punctured balls in manifolds

Shigeru Itoh, A random fixed point theorem for a multivalued contraction

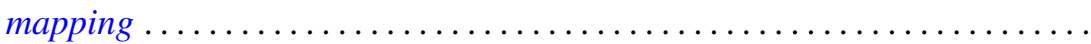

Nicolas P. Jewell, Continuity of module and higher derivations . . . . . . ......

Roger Dale Konyndyk, Residually central wreath products . . . . . . . . . . .

Linda M. Lesniak and John A. Roberts, On Ramsey theory and graphical

parameters.

Vo Thanh Liem, Some cellular subsets of the spheres.

Dieter Lutz, A perturbation theorem for spectral operators

P. H. Maserick, Moments of measures on convex bodies ... . . .

Stephen Joseph McAdam, Unmixed 2-dimensional local domains . .

D. B. McAlister and Norman R. Reilly, E-unitary covers for inverse semigroups...

William H. Meeks, III and Julie Patrusky, Representing codimension-one

homology classes by embedded submanifolds . . .

Premalata Mohapatro, Generalised quasi-Nörlund summability . .

Takahiko Nakazi, Superalgebras of weak-*Dirichlet algebras .

Catherine Louise Olsen, Norms of compact perturbations of operators .

William Henry Ruckle, Absolutely divergent series and isomorphism of

subspaces. II.

Bernard Russo, On the Hausdorff-Young theorem for integral operators .

Arthur Argyle Sagle and J. R. Schumi, Anti-commutative algebras and

homogeneous spaces with multiplications ............

Robert Evert Stong, Stiefel-Whitney classes of manifolds .

D. Suryanarayana, On a theorem of Apostol concerning Möbius functions of

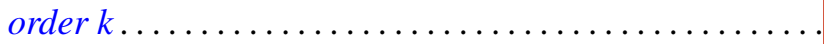

Yoshio Tanaka, On closedness of $C$ - and $C^{*}$-embeddings . . 